\title{
Chaos, Turbulence and Fractal: Theory and Applications
}

\author{
Edgar E. Escultura \\ Professor V. Lakshmikantham-GVP Institute for Advanced Studies, GVP College of Engineering, J. Nehru Technical University \\ Kakinada, Vishakhapatnan, AP, India \\ Kakinada, India \\ Email: escultur36@gmail.com
}

Received January 4, 2013; revised April 5, 2013; accepted April 12, 2013

Copyright (C) 2013 Edgar E. Escultura. This is an open access article distributed under the Creative Commons Attribution License, which permits unrestricted use, distribution, and reproduction in any medium, provided the original work is properly cited.

\begin{abstract}
The paper identifies chaos, turbulence and fractal of quantum and macro gravity and studies their behavior, properties and applications based on the grand unified theory (GUT) and qualitative mathematics and modeling. Applications include devising electromagnetic engines, tornado aborter and terminator and technologies for electromagnetic treatment of genetic diseases such as cancer, systemic lupos erythematosus, diabetes and mental disorder without harm to normal cell and side effect. Typhoon in the Western Pacific which is turbulence is impossible to terminate and impractical to deflect but prediction can be improved because it follows the Northern Pacific Wind Cycle (Southern Pacific Wind Cycle in the Southern Hemisphere) and is affected by the temperature variation over the Philippine Deep and around Mayon Volcano. The electromagnetic engine uses the clean, inexhaustible, free dark matter, specifically, the energy of magnetic flux, in place of conventional fuel, e.g., fossil, nuclear and geothermal. The tornado aborter and terminator utilize the gravitational flux of the Earth, its vortex flux of superstrings as a cosmological vortex which is turbulence. The technologies for electromagnetic treatment of genetic diseases utilize electromagnetic waves based on resonance. All of them are GUT technologies because they are applications of GUT. Except for the magnetic train which is in operation the rest is still at the conceptual and research and development (R\&D) phase but the theory is complete and the strategies for R\&D are laid down in detail in the cited original papers.
\end{abstract}

Keywords: Chaos; Cosmological Vortex; Dark Matter; Electromagnetic Engine; Electromagnetic Treatment of Genetic Diseases; Fractal; Gravitational Flux; Magnetic Flux; Prima; Quantum and Macro Gravity; Superstring; Toroidal Flux; Turbulence

\section{Introduction}

This paper focuses on the three nonlinear natural phenomena of chaos, turbulence and fractal. Chaos and turbulence are two of the three phases of the standard dynamics [1,2]; some cases of turbulence are fractal. Nonlinear analysis and phenomena cover very broad fields of mathematics and science which are rapidly expanding. While there is considerable treatment of the physical concepts chaos, turbulence and fractal in the scientific literature (e.g., [1-6]), it is less than adequate for purposes of precise scientific analysis. Moreover, they do not capture their intuitive sense and chaos, particularly, even not well defined. For example, chaos connotes disorder which is not amenable to computation. Therefore, the present methodology of quantitative modeling $[7,8]$ (formerly called mathematical modeling) that describes appearances of nature and natural phenomena mathematically and relies mainly on computation and measurement is inadequate and has left long standing problems of physics unsolved, e.g., the 200-year-old gravitational n-body problem [9], and outstanding issues such as the basic constituent of matter, unresolved. Furthermore, it is known that over $90 \%$ of matter in the Cosmos is dark [10] (now updated to over 95\% [11]), one of the two fundamental states of matter which is not observable and known only by its impact on visible or ordinary matter, the other fundamental state [12]. Thus, we need qualitative mathematics and modeling (QMAM), a new methodology formerly called dynamic modeling $[13,14]$ introduced in and the main contribution of [15] that explains appearances of nature and natural phenomena in terms of natural laws. It was applied to physics for the first time to solve the gravitational n-body turbulence 1997 [9] and, later, the turbulence problem, 2001 [1]. In fact, this new methodology has given rise to over 60 papers and books [16] in mathematics, physics and related 
fields that span biology [17], atmospheric and geological sciences [1], physical psychology [18] and medicine [19].

\section{The New Methodology}

Qualitative mathematics, the mathematical component of this new methodology is the complement of computation and measurement and consists of the following daily activity of the mathematician or scientist:

Making conclusions, visualizing, abstracting, thought experimenting, learning, creative activity, intuition, imagination, trial and error to sift out what is appropriate, negating what is known to gain insights into the unknown, altering premises to draw out new conclusions, thinking backwards, finding basic premises for a mathematical space and devising useful techniques.

It includes abstract mathematical spaces and the search for the laws of nature. QMAM is the only tool and method available for creating physical theory, i.e., mathematical space whose basic premises or axioms are laws of nature. Among the recent applications based on qualitative mathematics are: 1) the critique-rectification of the real number system that led to its development as the new real number system [20] and, through the latter, 2) resolution of the 360-year-old Fermat's last theorem $[21,22]$ that proved it false by way of counterexamples, 3 ) proof of the 250-year-old Goldbach's conjecture [21-23] and, combined with qualitative modeling, 4) the solution of the gravitational n-body and turbulence problems $[1,9]$ and development of the grand unified theory (GUT) that unifies natural science [7].

\section{The Superstring}

We distinguish physical from abstract concept because ambiguity on this matter leads to error, e.g., error of relativity theory in treating time as physical concept. A physical concept has a referent in the real world such as body or event. An abstract concept, e.g., mathematical concept, is a creation of the mind and neither exists in the real world nor does it have a physical referent, e.g., time and distance which describe relationships between events and objects, respectively $[8,18]$. The most fundamental physical concept is the superstring, basic constituent or fundamental building block of matter [7,11].

Mass is a measure of amount of matter in a physical system; in the metric system a unit of mass is the kilogram. Energy is motion of matter; therefore, mass and energy are never separate and neither pure matter nor pure energy exists. Therefore, every piece of matter has energy and anything that has energy is matter. For example, the photon has mass $[7,8,11]$. Wave is synchronized vibration of its medium which does not go with the wave. In water wave every molecule in its path vibrates but does not go with it. Basic cosmic or electromagnetic wave is generated by the normal vibration of atomic nucleus and propagated across dark matter as suitably synchronized vibration of the superstrings that comprise it. Nuclear vibration is due to the impact of cosmic waves coming from all directions in the Cosmos [8,24]. Electromagnetic wave is fractal which accounts for its huge energy and the only force in the Cosmos that interacts with, agitates and breaks or converts a superstring from one form to another, dark or visible $[11,24]$.

Another type of cosmic wave is seismic wave (profile in Figure 7 of [24]) generated by the micro component of turbulence at its interface [1]. Such interface is found between adjacent layers of the spinning core of a cosmological vortex, volcanic lava flow in a volcano and at conservative tectonic plate boundary. They convert dark to visible matter in the interior of a cosmological vortex in the Cosmos, around volcano and along geological fault and tectonic plate boundary $[1,25]$. In the Cosmos the appearance of cosmic dust that congeals into cosmological vortices at the rate of one star per minute is sustained by seismic waves generated by spinning cores of cosmological vortices [25]. Seismic waves are generated at lava flow and geological fault that produce balls of fire around them $[1,2]$. They are also generated by lightning in the lower atmosphere that produces earthlights in the upper mesosphere $[25,26]$. (For detail, see $[1,13]$ ). Flux is motion of matter with identifiable direction at each point. Turbulence is coherent flux, i.e., having no opposing motion except in a synchronized manner. Among the examples of flux are water current, the atom, cosmological vortex, typhoon, magnetic flux and electromagnetic wave which are turbulence. A vortex flux spins around its eye which is a region of calm and low pressure that sucks matter around it. Some of these concepts are tentative pending their precise definition by the laws of nature. We take the most fundamental natural law of GUT [7]:

Energy Conservation. In any physical system and its interaction, the sum of kinetic (visible) and latent (dark) energy is constant, gain of energy is maximal and loss of energy is minimal.

This is an extension and enrichment of the first law of thermodynamics.

Energy Conservation Equivalence. Its various expressions are: Energy conservation has many expressions or forms: order, symmetry, economy, least action, optimality, efficiency, stability, self-similarity (nested fractal), coherence, resonance, quantization, synchronization, smoothness, uniformity, motion-symmetry balance, non-redundancy, non-extravagance, evolution to infinitesimal configuration, helical and related configuration such as circular, spiral, sinusoidal, the tendency to form a loop and, in biology, genetic encoding of characteristics, reproduction and order in diversity and complexity of functions and configuration that provides op- 
timal capability.

This law is instrumental in deciding scientific issues. For instance, physicists were excited by the discovery of the third quark in 2004 which they thought was the gluon that supposedly binds two protons in the narrow confines of the nucleus. It follows from the principles of nonextravagance and non-redundancy [11] that the third quark is really the -quark for it joins the two +quarks to form the proton just as the third quark joins two positive quarks one from each of two protons [12,24]. In other words, nature does not create or keep two physical systems with the same function or create a physical system beyond what it needs. The next law is central to turbulence and applies to dark matter, gas and liquid

Flux-Low-Pressure Complementarity. Low pressure sucks matter around it and the initial rush of matter towards a region of low pressure stabilizes into local or global coherent flux called turbulence; conversely, coherent flux induces low pressure around it.

Hit by suitable electromagnetic wave, a non-agitated superstring (a) is thrown by its impact, bounces with other superstrings and comes to rest in dark matter when its imparted energy is dissipated or (b) it gets close to its earlier path, gets sucked by it, by Flux-Low-Pressure Complementarity, and forms a loop, the original nonagitated superstring called toroidal flux travelling through the loop at $7 \times 10^{22} \mathrm{~cm} / \mathrm{sec}$ [27]. By Energy Conservation and Energy Conservation Equivalence, the loop shrinks and evolves into its most energy-conserving configuretion, namely, circular helical loop, its toroidal flux traveling through its cycles at the speed of $7 \times 10^{22} \mathrm{~cm} / \mathrm{sec}$. By the fractal principle its toroidal flux, being a superstring, has toroidal flux, a superstring, traveling at this speed, etc., leading to formation of nested fractal sequence of toroidal fluxes, each a superstring. The first term is a semi-agitated superstring identified with the fractal superstring since it alone interacts with cosmic waves and other superstrings in its neighborhood. By the quantization and synchronization principles of the Energy Conservation Equivalence Law, this speed of $7 \times$ $10^{22} \mathrm{~cm} / \mathrm{sec}$ is a constant of nature.

We articulate our findings as natural law.

Existence of Basic Constituent of Matter and its Generalized Nested Fractal Structure. The basic constituent of dark matter is the non-agitated superstring. It is a circular helical loop and nested fractal sequence of superstrings or toroidal fluxes, with itself as first term; each toroidal flux in the sequence is a superstring having toroidal flux, a superstring, traveling at $7 \times 10^{22} \mathrm{~cm} / \mathrm{sec}$ along its cycles, etc.; each superstring except the first, is contained in and self-similar to the preceding term in structure, behavior and properties.

This structure called nested generalized fractal [28] is an extension of geometrical fractal $[3,6]$. Since the su- perstring is a physical concept it is a nested generalized physical fractal. Its self-similar general property is its helical structure with the toroidal flux (a superstring) traveling through its cycles at $7\left(10^{22}\right) \mathrm{cm} / \mathrm{sec}$. A superstring is non-agitated (dark) if its cycle length (CL) is less than $10^{-16}$ meters, semi-agitated if $10^{-16}<\mathrm{CL}<10^{-14}$ meters and agitated (visible) if a segment has CL $>10^{-14}$ meters (Figure 1 of [24] shows a semi-agitated superstring).

In the agitation or semi-agitation of the superstring there is another possibility: (c) hit by suitable electromagnetic wave the first term of a non-agitated superstring expands and becomes a semi-agitated superstring. In all these cases the superstring is nested generalized physical fractal sequence of superstrings.

It is the nested generalized physical fractal structure that makes the superstring indestructible and the Universe of dark matter timeless, i.e. having no beginning and no end, since electromagnetic wave may only resonate with and break a finite number of toroidal fluxes. Moreover, by Flux-Low-Pressure Complementarity, the Universe of dark matter is boundless. Our universe is a local bubble in it with specific birth and destiny [25].

\section{The Primum}

The energy of the superstring is enshrined in its toroidal fluxes. The first term of its fractal sequence looks like a lady's spring bracelet (see figure in [29]). This fractal structure of the superstring not only makes it indestructible but also packs huge energy in it and, therefore, dark matter as well. The latent energy density of dark matter, part of which convertible to kinetic energy, is $10^{26}$ joules/ cubic $\mathrm{ft}$ according to de Broglie [30], $8\left(10^{8}\right)$ volts $/ \mathrm{cm}$ says Seike Jr. [30] and the equivalent of $18 \mathrm{~kg} / \mathrm{cu}$ meter according to Gerlovin [31] using relativistic conversion. This convertible energy is only a small portion of the total energy density of dark matter.

Possibilities (a) and (b) may occur only when electromagnetic wave hits a non-agitated superstring. However, when it hits a semi-agitated superstring a pair of mutually exclusive possibilities may occur: (1) a segment of the first term of the nested fractal superstring in (c) bulges to retain the speed of the toroidal flux despite the energy imparted by the electromagnetic wave that hits it, by Energy Conservation, turning it into an agitated superstring called primum, unit of visible matter, its toroidal flux non-agitated, or (2) the first term breaks, its toroidal flux remaining non-agitated and dark superstring.

Dark-to-Visible-Matter Conversion. When suitable electromagnetic wave hits a semi-agitated superstring one and only one of these occurs: (a) the outer superstring breaks, its toroidal flux remaining non-agitated and (b) a segment bulges into a primum, an agitated su- 
perstring and a unit of visible matter.

The indestructibility of the superstring follows from the Resonance Law discovered in the course of explaining the disastrous final flight of the Columbia Space Shuttle $[32,33]$ since suitable electromagnetic wave hits and resonates only with the first term of the fractal sequence leaving the tail fractal sequence intact as a superstring in accordance with this law:

Resonance Law. Maximum resonance between waves, oscillation or vibration occurs when they have exactly the same characteristics with wavelength or frequency as the principal factor. The degree of resonance declines with the difference between wave characteristics However, at suitably high order of magnitude of wavelength the infinitesimal effect of resonance with orders of magnitude nearby rises to significance.

The disastrous final flight of the Columbia Space Shuttle is attributed to this law. A physical system is observable through the medium of light if its size is comparable with the wavelength of the latter; this follows from the Resonance Law. Thus, a semi- or non-agitated superstring is not observable, i.e., dark, because its cycle length is less than the finest wavelength of visible light in order of magnitude, $10^{-14}$ meters.

We define chaos precisely and identify the emergence of chaos, turbulence and fractal associated with the prima. Chaos is a mixture of order none of which is identifiable (note the contrast with turbulence). This is consistent with order defined by natural laws since every piece of matter is subject to them. Chaos is linked to the uncertainty of large and small number [20]. For example, it is impossible to identify the position of an electron in flight in an interval of time because of its great speed (large number) and minute size (small number). This uncertainty is expressed by the Heisenberg uncertainty princeple [31] that says it is impossible to simultaneously pinpoint the position and velocity of an electron in flight. In other words, the path of the electron is chaos. Chaos gives way to turbulence when the electron breaks and its toroidal flux rests in dark matter or its energy dissipates and reduces to a non-agitated superstring which is dark turbulence.

When the primum's toroidal flux, i.e., the second term of its fractal sequence, is hit by electromagnetic waves coming from all directions it is thrown into erratic motion, collides with other superstrings and, combined with steady hits by electromagnetic waves coming from all directions, turns it into a spike with the centroid traveling through the cycles at $7\left(10^{22}\right) \mathrm{cm} / \mathrm{sec}$. This configuration and motion pulls the superstrings around the primum into its induced vortex flux with axis coinciding with the center of its cylindrical eye. This makes the primum a magnet, its polarity in accordance with the right hand rule of electromagnetism [24]. The induced vortex flux is its magnetic flux which is turbulence. Viewed from its north pole, the induced vortex flux is counterclockwise if it is a positive primum, by convention. It is negative otherwise. The induced vortex flux is dark but its impact on visible matter is its charge. The unit of charge by convention is the electron's charge which is $-1\left(1.6 \times 10^{-19}\right.$ coulombs $[12,24])$. The electron is a basic primum; the other basic prima are: +quark, charge $+2 / 3$ and -quark, charge $-1 / 3$, basic because they comprise every atom [28]. They are produced at rapid rate in the Cosmos and cellular membrane of living things, in the latter to support their growth [17,25].

The spike itself is chaos but as soon as the induced vortex flux forms, it shields it from the impact of cosmic waves and becomes part of the turbulence that is the vortex flux. The vortex flux ultimately loses energy and the superstring becomes non-agitated. Thus, the primum that starts as chaos becomes turbulence then non-agitated superstring which is dark turbulence that belongs to the standard dynamics [1].

\subsection{Primal Interaction}

Primal interaction is governed by Flux-Low-pressure complementarity and the next law.

Flux Compatibility. Two prima of opposite toroidal flux spins attract at their equators but repel at their poles; otherwise, they repel at their equators but attract at their poles. Two prima of same toroidal flux spin connect equatorially only through a primum of opposite toroidal flux spin between them called connector.

The primum's profile is sinusoidal and the trace of the maximum point of its induced vortex flux is the equator [24]. The proton is a pair of +quarks joined by a -quark equatorially $[12,24]$, by Flux Compatibility (Figure 3.3 of [24]). By Energy Conservation, their axis are coplanar; its charge: $2 / 3-1 / 3+1 / 3=+1$. Thus, there is net coherent counterclockwise vortex flux of charge +1 around the proton. We note the transition phase of chaos as the fluxes from the two +quarks collide until they stabilize when the vortex flux of the -quark becomes an eddy in it. Since simple primum, e.g., positron, is charged, the neutral neutrino must be a coupled pair of simple prima of numerically equal but opposite charges, say, $+q$ and $-q$, so that its charge is $+q+-q=0$, neutral [12].

By Flux Compatibility, the electron can attach itself to a positive quark of the proton at any point but Energy Conservation and the optimality principle of the Energy Conservation Equivalence Law require that it attach to both +quarks beside the negative quark as the most stable position but pushes the negative quark a bit, by Flux Compatibility, so that their centers viewed from the north pole form the vertices of a quadrilateral. In its interior are the coherent vortex fluxes of the positive quarks, nega- 
tive quark and electron that make it a region of low pressure called depression. By Flux-Low-Pressure Complementarity its interior sucks neutral primum around it since charged primum is repelled by primum of the same charge already in the coupling. Therefore, only suitably light neutral primum fits in and that is the neutrino. We have just composed the neutron consisting of a proton, electron and neutrino. Its charge is: $+2 / 3-1 / 3+2 / 3-1$ $+0=0$, i.e., neutral, and there is no net coherent vortex flux around it. The vortex flux of a charged coupled primum is also discular for the same reason that the simple primum's vortex flux is due to greater centrifugal force along the equatorial plane.

We note that the initial coupling of the proton is chaos due to the collision of the vortex fluxes of the +quarks until the -quark becomes an eddy in their combined flux.

Since the masses of the neutron, proton and electron are known: $1.0087 \mathrm{amu}, 1.0073 \mathrm{amu}$ and (5.486)10 ${ }^{-8}$ amu, respectively $[12,24]$, the mass of the neutrino is $\eta=$ $8.5 \times 10^{-8} \mathrm{amu}\left(1 / 1250^{\text {th }}\right.$ proton's mass); ironically, neutrino's mass is still a subject of hot pursuit [34-36]).

\subsection{The Atom}

The nucleus forms first in the atom and consists of protons alone (see figure in [29]), except the hydrogen atom, joined together by -quarks. Their toroidal fluxes add up to a coherent discular vortex flux whose eye contains the nucleus. So do their charges add up to the charge of the atom, i.e., equal to the number of protons in the nucleus. However, the nucleus itself is neutral since the protons' induced toroidal fluxes cancel each other. As coupled primum the nucleus is a magnet of positive polarity with the vortex flux around it its magnetic field. From the right-hand rule with the index finger pointing in the direction of the vortex flux, the thumb points to the north-pole. Viewed from its north-pole the vortex flux of a free atom spins counterclockwise so that by Newton's action-reaction law the nucleus rotates in the opposite direction as a unit. This is the spin of quantum physics distinct from the toroidal flux spin. It is the nested fractal structure of the nucleus being comprised of fractal prima that makes the electromagnetic wave it generates fractal.

Electrons being negatively charged are attracted to the vortex flux away from the eye, by Flux Compatibility and Flux-Low-Pressure Complementarity. However, being light, they are swept into orbit by the vortex flux and become orbital electrons, their orbits probabilistically symmetrical (explained later) with respect to the equatorial plane. By centrifugal force, the most energetic orbital electrons are those closest to the equatorial plane. They form the outermost orbital shells. The least energetic ones cluster near the poles and form the lowest orbital shell. A stable atom has orbital electrons equal in number to the protons in the nucleus. Otherwise, it is a positive ion when there is a deficiency in orbital electrons or negative when there are more orbital electrons than protons. Moreover, by Flux-Low-Pressure Complementarity, non-agitated superstrings accumulate steadily in the center of the nucleus, the principal source of energy in nuclear fission. Charged prima are repelled by the +quarks and -quarks. However, the neutron being neutral may be sucked by the nucleus, by Flux-LowPressure Complementarity, to form a heavy isotope. As primal coupling, transitional chaos occurs in the formation of the nucleus but permanent chaos in the orbital electrons (during the life of the atom) due to the effect of the Heisenberg Uncertainty Principle [37] and bombardment by cosmic waves. That is why the electron's orbit is not an ordinary curve but a probability curve. It is in this curve where the probability of finding an electron is greatest. This chaos reduces to turbulence as the proton loses its kinetic energy and becomes a cluster of nonagitated superstrings at the terminal phase of the socalled neutron star [25].

There is a lingering chaos, an infinitesimal spike due to the impact of cosmic waves that hit and resonate with electromagnetic wave. In fact, the existence of such infinitesimal spike is true of all vortex fluxes of superstrings for the same reason. The turbulence of the electromagnetic wave continues until its kinetic energy is dissipated; then the electromagnetic wave vanishes and ceases to vibrate the superstrings along its path.

When orbital electrons are expelled by turbulence or cosmic waves the atom becomes a positive ion. It can also become negative ion when more electrons are drawn into orbit by Flux-Low-Pressure Complementarity in addition to Flux Compatibility. Two valence electrons (of the same or different elements) are joined by a -quark to form a molecule. Stable molecule share a pair of valence electrons and gas atoms generally exist as di-atoms (coupling of two atoms) for maximal stability.

\subsection{Primal Polarity}

The Earth is a cosmological body its vortex flux of superstrings the gravitational flux. Its core is the collected mass around the eye including the atmosphere. Its gravitational flux shields Earth from massive approaching asteroids close to its equatorial plane and separates positive prima from their negative anti-matter that otherwise would create instability. However, there is no shield against an asteroid when it approaches either pole oblique to the equatorial plane; hence, the relatively frequent hits close to the North Pole. The Earth's gravitational flux goes from West to East, is fastest at the Equator and slows down towards 0 at the eye's extremities at the poles. Naturally, its pull on the Earth's material also 
slows down from the Equator to either Pole (polar lag) but the eye's suction is not diminished there. (The Sun's polar lag is $30 \%$ midway between the equator and either pole) Every cosmological vortex has a polar lag; this value $(30 \%)$ should be checked if it is a constant of nature. In Earth, the polar lag accounts for the counterclockwise spin of Earthly vortices, e.g., tornado and hurricane in the Northern Hemisphere (clockwise in the Southern Hemisphere) known as the carioles effect.

We look at the primum as it pops out of dark matter. Since the dark superstring has infinitesimal induced flux, it has no polarity and does not interact with anything except cosmic waves; it is oriented randomly. When a dark superstring is agitated by suitable cosmic wave and pops out of dark matter as free positive primum and its equatorial plane is oblique to the direction of the gravitational flux it rotates suitably due to the coriolis effect, its north pole pointing North and equatorial plane parallel to the Earth's equatorial plane (vortex flux spins from West to East) making its vortex flux a counterclockwise eddy in the gravitational flux, its optimal energy-conserving alignment. By Flux Compatibility, the positive primum is pushed up so that there is abundance of free positive prima in the upper atmosphere (confirmed by shower of fragments of protons smashed by ultra-energetic cosmic waves that hit Earth [38]). Free neutral prima are oriented randomly but free positive ions are counterclockwise eddies in the Earth's gravitational flux; they are pushed upwards, by Flux Compatibility. However, being heavy, they remain in the lower atmosphere. The free electron as clockwise eddy in the Earth's gravitational flux whose equatorial plane is parallel to the Earth's equatorial plane is pushed downwards, by Flux Compatibility. Thus, there is abundance of free electrons on the ground. Other free negative prima including the -quarks should be abundant on the ground also but we do not know where they are and no study has been done on them. It is possible that they are also carrier of electric current (moving charge) as the electron is. When the voltage between the positive ions in the lower atmosphere and the electrons on the ground reaches critical level they rush towards each other, collide and explode as lightning.

The separation between positive and negative prima contributes to the stability of cosmological vortices like Earth because it separates them from their anti-matter. Outside the Earth's gravitational flux primal orientation is determined by the dominant gravitational flux there; between planetary gravitational fluxes it is the Sun's gravitational flux, etc. There is no point in the Cosmos without some dominant gravitational flux.

Unsynchronized collision of physical systems as in chaos lacks coherence, is energy dissipating and evolves towards energy conserving configuration and motion. A familiar example of Earthly turbulence is the typhoon which is a vortex flux of air molecules [1]. It lasts until the temperature gradient between the warm and cold air along its path vanishes. Another example is a galaxy (including our universe as a super, super galaxy [25]) which is a vortex flux of superstrings (cosmological vortex) that lasts over a long, long time until its kinetic energy is completely dissipated, by Energy Conservation [7,25]. These examples belong to the standard dynamics [1] where a change in the normal conditions trigger the occurrence of chaos as transitional phase towards turbulence, by Energy Conservation, the latter vanishing when the normal conditions set in again.

\section{Macro Standard Dynamics}

We recall that the standard dynamics is a cycle that starts with normalcy like a calm summer day in the Pacific Ocean. Then some change in the conditions occurs that induces passage to the transitional and unstable phase of chaos. Energy Conservation induces its transition to turbulence until the change in the normal conditions that served as initial boundary conditions vanish and the cycle is completed as the conditions become normal.

\subsection{Earthly Turbulence}

We consider other turbulence rooted in dark matter.

\subsection{Wind Cycles}

The rotation (spin) of the Earth is due to the pull of the Earth by the gravitational flux. This pull is the effect of the combination of dark viscosity and resonance between the normal variation of the dark component of Earth's material and that of dark matter. Both the pull and the linear displacement are proportional to the density of the Earth's material. Since the Earth's crust is denser than that of, say, the Pacific Ocean there is a net flow of the Ocean which constitutes the Pacific Ocean Current going west. By Energy Conservation, the current finds the most energy conserving path, splits along the Equator off the Coast of the Asian mainland with the northern strip veering northeast and then east off the southern coast of South Korea and Japan, heads east south of Siberia, veers southeast west of the western seaboards of Canada and the US and veers southwest again to form a loop with the Equatorial current from East to West called the Northern Pacific Ocean Cycle [39] (see map in [40]).

We really do not need this cycle but we need the same analysis to map the Northern Pacific Wind Cycle [41]. Since the atmosphere is less dense than the Ocean there is air current that starts off the Coast of Ecuador going west called the Trade Winds used by traders in the past to cross the Pacific Ocean along the Equator. It goes west 
into the Western Pacific, splits off east of the eastern Philippines Coast the northern strip veering north then northeast scraping Vietnam and Southern China, crossing North and South Korea and Japan and becoming the jet flow that airlines use going east to save on fuel. Then it veers south east of Siberia and across the Bearing Sea as it crosses Alaska, veers southeast as it crosses Canada and south into the Tornado Belt in the US $[1,2,39,41]$. It veers southwest then west and joins the Trade Winds off the coast of Ecuador to complete the Northern Pacific Wind Cycle (map in [41]). Both the Northern Pacific Ocean Current and the Northern Pacific Wind Cycle are vortices, i.e., turbulence with clockwise spins not on account of Flux Low Pressure Complementarity alone but combined with the land mass distribution and the tendency to form a loop. The latter wind cycle plays a major role in typhoon and tornado. They have mirror images in the southern Pacific with counter clockwise spins (maps in $[40,41])$.

\subsection{Typhoon and Tornado}

We first look at the conditions that give rise to a typhoon known as hurricane in the Western Hemisphere. The Earth is studded with under-ocean volcanoes, especially, along conservative plate boundaries called trenches. We consider the Marianas Trench in the Western Pacific about 1500 miles east of the Philippines above the Equator. Active volcanoes along the Trench erupt periodically, heat up the ocean surface and produce pockets of warm water called el niño. When the pockets are sufficiently close together and broad or solid, say, as broad as the Australian landmass, the el niño heats up the lower atmosphere sufficiently to create a tropical depression (low pressure). The depression sucks air around it, by Flux-Low-Pressure Complementarity and the initial rush and ensuing collision of trillions of air molecules create chaos that, by Energy Conservation, gives way to a typhoon, a vortex flux of air molecules which is turbulence (details in $[1,39]$ ) that spins counterclockwise due to the coriolis effect (just as water eddy in a spring spins counterclockwise when there is a relative counter current from west to east and the current is flowing eastward. This is quite analogous to the north polar lag in the Earth's core $[1,39]$. The typhoon follows the Northern Pacific Wind Cycle but is affected by the temperature variation over the Philippine Deep Trench and around Mayon Volcano $[1,39,40]$. This information allows precise prediction of the course of a typhoon in this region. Typhoon can be deflected but that would be impractical. Another destructive Earthly turbulence that belongs to the standard dynamics is the tornado. We look at the Tornado Belt in the US, a 1000- by 650-mile rectangular region that extends from Oklahoma to Florida and from the Midwest to Texas.

The Northern Pacific Wind Cycle interfaces with the narrower Northern Atlantic Wind Cycle over the Belt. The latter has the same spin as the Northern Pacific Wind Cycle but opposite fluxes at the interface. The interface wobbles between the western and eastern edge of the Belt during spring as eddies form on both sides of the interface like ball bearings that facilitate the spin, by Energy Conservation. The cycles press against each other, by Flux Compatibility.

During spring, the Northern Pacific Wind Cycle pulls the cold air from the Arctic, by Flux-Low-Pressure Complementarity, and brings it over the Tornado Belt at the interface between the Cycles. At the same time, the Northern Atlantic Wind Cycle brings the warm air from the Equator, especially, the Caribbean, over the Belt also at the interface. The warm and cold air pressing against each other, by Flux Compatibility, forming a sharp interface between them thrusting the lighter warm air upwards and the denser cold air downwards and creating a horizontal cylindrical vortex flux of air that when its northern end tilts downwards (as the energy-conserving motion in the Northern Hemisphere due to the coriolis effect) and touches and sucks the ground, it forms the funnel of a full tornado (details in [1,39]). Minor tornadoes also form along the interfaces of eddies on both sides of the interface.

We refer the reader to $[1,39]$ for discussion of earthquake as Earthly turbulence belonging to the standard dynamics. Since the destiny of our universe is a cluster of black holes [25] which are turbulence. Thus, all turbulence is the final phase of the standard dynamics.

\subsection{Astronomical Turbulence}

Every explosion creates chaos and previous order and history and every chaos evolves to turbulence; then turbulence vanishes back into normal conditions and completes the cycle of the standard dynamics. The Big Bang $[13,42]$ which was the explosion of a super, super massive black hole 8 billion years ago [7,25] and the destiny of the core of a previous universe $[8,25]$, created a super, super depression in dark matter. The explosion created an expanding wave front as the boundary of the super, super depression called the Cosmic Sphere [8,25]. At $t=1.5$ billion years from the initial burst of the Big Bang, the Cosmic Sphere burst $[8,25,43]$ releasing the trapped semi-agitated superstring that formed the early prima which clustered as the early visible matter of our universe called quasars that peaked at $\mathrm{t}=2.5$ billion years [39]. We put the birth of our universe at Cosmic Burst. The quasars got absorbed by the galaxies in the neighborhood of the once Cosmic Sphere [25,44]. Neither the Big Bang nor the Cosmic Burst created new 
galaxies but as the super, super depression gave way to a super, super cosmological vortex that expanded and evolved to the present super, super galaxy, our universe, it absorbed galaxies formed before the Big Bang as new galaxies emerged steadily [25]. In fact, the Milky Way is one of those that formed earlier as it has stars older than the Big Bang [45]. All the galaxies of our universe including the super, super galaxy are turbulence belonging to the standard dynamics. They will vanish back into dark matter as black holes, their destiny consisting of massive concentration of non-agitated superstrings in the eyes of the once cosmological vortices, and complete the standard dynamics. In effect, all turbulence belongs to the standard dynamics since all visible matter ultimately become dark (For details of the cosmology of our universe, see [13,25]).

\section{Applications}

For practical applications of the theory of turbulence we refer the reader to the original papers on GUT and its theoretical and practical applications to the development of technologies that utilize dark matter instead of conventional fuel and power, particularly, the magnetic flux as natural engine instead of gasoline or coal-fired power generator, nuclear reactor, geothermal plant, hydroelectric power plant and other conventional power generator $[19,46]$. They include the electromagnetic engine [19] that powers the magnetic train, electric power generators, cars and other motor vehicles, aircraft and space vehicle. We call them type I GUT technology. This type relies on the properties of the magnetic flux which is dark turbulence and functions in accordance with Flux Compatibility and Flux-Low-Pressure Complementarity. Except for the electromagnetic engine that powers the electromagnetic train and is in operation, the rest is at the research and development phase with the exception of the one for spacecraft and space vehicle which is still long-term and at the conceptual phase although the theory is complete.

Another type of GUT technology (type II) consists of the tornado aborter and terminator or deflector [39]. (Since this technology is still at the research and development phase, details are omitted). Tornado always starts at the interface of warm and cold air about $1.5 \mathrm{~km}$ above ground. The aborter blurs the interface between the nascent tornado and the ground by warm air to prevent its touchdown. When it fails to touchdown in seconds it vanishes. In the case of a full tornado traveling along the interface (always in the northerly direction except those along an eddy), the terminator works on the same principle as the aborter except that it is applied at its forward path on the ground along the interface to blur it and the temperature gradient between the interfacing cold and warm air to break the tornado or divert it around the city being protected. This type utilizes the gravitational flux which is turbulence based on Flux Compatibility and Flux-Low-Pressure Complementarity.

The third type uses electromagnetic waves which are turbulence for electromagnetic treatment of genetic diseases such as cancer, systemic lupos erythematosus, diabetes, muscular dystrophy and mental disorder [17].

The fourth type is GUT technology based on the theory of intelligence [18] that includes what we call a social technology for (1) devising a program of mathematics-science education from primary through graduate school $[8,47,48]$ based on [18] and (2) devising a strategy for sustainable economic-industrial development of the Third World involving complex systems [49,50].

We focus on genetic treatment [19] the underlying theory which is also complete and research and development involves devising the appropriate technology for each of these diseases. The treatment does not harm normal cells and, therefore, has no side effect; it is based mainly on the Resonance Law and [17,51]. We summarize the needed key information for this class of technologies.

(a) Brain waves, electromagnetic waves encoded with vibration characteristics of a living cell, e.g., neuron, gene, are the brain's and gene's medium for carrying out their functions [17-19].

(b) The neutralizer gene emits brain waves that create its mirror image in the chromosome, in effect, inserting itself in the DNA sequence that alters the gene and removes the undesirable symptom of a disease [17,19]. This technology is now used for treatment of cystic fibrosis by introducing a neutralizer gene into the lung that modifies its gene suitably. Now DNA can be introduced into the body to modify or alter the gene of any genetic disease that determines its undesirable symptom suitably. The stem cell belongs to this class of technology.

(c) A technology can be devised that projects radiation that resonates only with a defective gene. The radiation intensity can be raised suitably to sterilize the gene or even destroy it; in the former the cell will not be reproduced in the normal turnover of cells.

(d) The unified theory of evolution [17] lays down physical therapeutic procedure based on the Genetic Encoding Law [17] for reversing, not just arresting, the impact of degenerative diseases such as muscular dystrophy and controlling some chronic mental disease such as autism. This procedure is also the basis for training athletes by providing them with appropriate reflexes and physical skills since both of them are genetic [17].

(e) Certain new mental activity such as creative and new physical activity, e.g., standing on one foot and new exercises, raises neural interconnections that counter the impact of aging like narrowing of the brain [18].

(f) Through emission of brain waves the gene converts 
superstrings to basic prima in the cellular membrane of plants and animals that form their tissues [17,52-54].

Finally, collaborative research and development of appropriate technology for electromagnetic treatment of these diseases will require participation of experts in GUT, the genetic diseases concerned who can identify the neutralizing gene for each or its distinguishing vibration characteristics, especially, wave length; a nuclear engineer who can identify suitable radioactive material to provide the required resonant radiation, if any, and radiation engineer who can design and devise appropriate technology to emit desired resonant radiation for each of these diseases. Since cystic fibrosis has already known neutralizer we can focus on genetic composition and sterilization of other genetic diseases.

Nonlinear, in the words of Prof. V. Lakshmikantham, Founder of Nonlinear Analysis, is not necessarily linear which means that all natural phenomena are nonlinear and at the micro scale of existence nothing is linear. Chaos is a complex system $[39,49]$ and by Energy Conservation a transitory phase of turbulence whose limit is the epitome of order-dark matter.

\section{REFERENCES}

[1] E. E. Escultura, "Turbulence: Theory, Verification and Applications," Nonlinear Analysis, A-Series: Theory, Methods and Applications (TMA), Vol. 47, No. 8, 2001, pp. 5955-5966.

[2] E. E. Escultura, "Earthly Turbulence," In: E. E. Escultura, Ed., Scientific Natural Philosophy, Bentham Science Publishers, 2011, pp. 237-260

http://www.benthamscience.com/eBooks/9781608051786 /index.htm

[3] G. A. Edgar, "Measure, Topology and Fractal Geometry," Springer-Verlag, New York, 1990. doi:10.1007/978-1-4757-4134-6

[4] J. Gleick, "Chaos: Making a New Science," Penguin Books, New York, 1988.

[5] C. A. Pickover, "Chaos and Fractals," Elsevier Science, Amsterdam, 1998.

[6] 6K. J. Falconer, "The Geometry of Fractal Sets," Cambridge University Press, Cambridge, 1986.

[7] E. E. Escultura, "The Grand Unified Theory," Nonlinear Analysis, A-Series: TMA, Vol. 69, No. 3, 2008, pp. 823831. doi:10.1016/j.na.2008.02.043

[8] E. E. Escultura, "Qualitative Mathematics and Modeling: Theoretical and Practical Applications," LAP LAMBERT Academic Publishing, in press.

[9] E. E. Escultura, "The Solution of the Gravitational nBody Problem," Nonlinear Analysis, A-Series: TMA, Vol. 30, No. 8, 1997, pp. 5021-5032.

[10] P. C. W. Davies and J. Brown, "Superstring, A Theory of Everything," Cambridge University Press, New York, 1988.

[11] E. E. Escultura, "The Mathematics of the Grand Unified
Theory," Nonlinear Analysis, A-Series: TMA, Vol. 71, 2009, pp. e420-e431.

[12] E. E. Escultura, "Qualitative Model of the Atom, Its Components and Origin in the Early Universe," Nonlinear Analysis: Real World Applications, Vol. 11, 2009, pp. 29-38.

[13] E. E. Escultura, "Dynamic Modeling of Chaos and Turbulence," Nonlinear Analysis, A-Series: TMA, Vol. 63, No. 5-7, 2005, pp. e519-e532.

[14] E. E. Escultura, "Superstring loop dynamics and applic to astronomy, biology," Nonlinear Analysis, A-Series: TMA, Vol. 35, No. 8, 1999, pp. 259-285.

[15] E. E. Escultura, "The Trajectories, Reachable Set, Minimal Levels and Chain of Trajectories of a Control System," Ph. D. Thesis, University of Wisconsin, Madison, 1970.

[16] E. E. Escultura, "Abstracts and Summary of Publications," The Grand Unified Theory.

http://users.tpg.com.au/pidro/

[17] E. Escultura, "The Unified Theory of Evolution," In: E. E. Escultura, Ed., Scientific Natural Philosophy, Bentham Science Publishers, 2011, pp. 237-260.

http://www.benthamscience.com/eBooks/9781608051786 /index.htm

[18] E. E. Escultura, "The Physics of Intelligence," Journal of Education and Learning, Vol. 1, No. 12, 2012, pp. 51-64.

[19] E. E. Escultura, "Electromagnetic Treatment of Genetic Diseases," Journal of Biomaterials and Nanobiotechnology, Vol. 3, No. 2, 2012, pp. 292-300. www.ccsenet.org doi:10.4236/jbnb.2012.322036

[20] E. E. Escultura, "The New Real Number System and Discrete Computation and Calculus," Neural, Parallel and Scientific Computations, Vol. 17, 2009, pp. 59-84.

[21] E. E. Escultura, "Exact Solutions of Fermat's Equation (Definitive Resolution of Fermat's Last Theorem)," Nonlinear Studies, 1997, Vol. 5, No. 2, pp. 227-2254.

[22] E. E. Escultura, "The New Mathematics and Physics," Journal of Applied Mathematics and Computation, Vol. 138, No. 1, 2003, pp. 127-149. doi:10.1016/S0096-3003(02)00126-1

[23] P. J. Davies, and R. Hersch, "The Mathematical Experience," Birkhäuser, Boston, 1981.

[24] E. E. Escultura, "Quantum Gravity,” In: E. E. Escultura, Ed., Scientific Natural Philosophy, Bentham Science Publishers, 2011, pp. 61-80.

http://www.benthamscience.com/eBooks/9781608051786 /index.htm

[25] E. E. Escultura, "Macro Gravity," In: E. E. Escultura, Ed., Scientific Natural Philosophy, Bentham Science Publishers, 2011, pp. 87-107.

http://www.benthamscience.com/eBooks/9781608051786 /index.htm

[26] D. Pendick, "Fires in the Sky," Earth, Vol. 20, 1996, pp. 62-64.

[27] V. A. Atsukovsky, "General Ether-Dynamics; Simulation of the Matter Structures and Fields on the Basis of the Ideas about the gas-Like Ether," Energoatomizdat, Mos- 
cow, 1990.

[28] E. E. Escultura, "Extended Geometrical and Generalized Fractal, Chaos and Applications," In: Kyle J. Brennan, Ed., Handbook on the Classification and Application of Fractals, Nova Publishers, 2011, pp. 1-39.

https://www.novapublishers.com/catalog/product_infinfo. php?products_id=23231

[29] E. E. Escultura, "From Macro to Quantum Gravity," Problems of Nonlinear Analysis in Engineering Systems, Vol. 7, No. 1, 2001, pp. 56-78.

[30] H. A. Nieper, "Revolution in Technology, Medicine, Society," Management Interessengemeinschaft für TachyonFeld-Energie, Odenburg, 1984.

[31] I. L. Gerlovin, "The Foundations of United Theory of Interactions in a Substance," Energoattomizdat, Leningrad, 1990.

[32] E. E. Escultura, "Columbia's Disastrous Final Flight: Crossroad for Science,” In: E. E. Escultura, Ed., Scientific Natural Philosophy, Bentham Science Publishers, 2011, pp. 81-87. doi:10.2174/97816080527071110101

[33] E. E. Escultura, "The Pillars of the New Physics and Some Updates," Nonlinear Studies, Vol. 14, No. 3, 2007, pp. 241-260.

[34] "Weighing in on Neutrino Mass," Science, Vol. 280, 1997, pp. 1689-1691.

[35] "Neutrinos Weigh in," Science, Vol. 282, No. 5397, 1998, pp. 2158-2159. doi:10.1126/science.282.5397.2158

[36] "Search for Neutrino Mass Is a Big Stretch for Three Labs," Science, Vol. 283, No. 5405, 1999, pp. 928-929.

[37] E. Merchbacher, "Quantum Mechanics," 2nd Edition, John Wiley \& Sons, Inc., New York, 1970.

[38] “Ultra-Energetic Particles," Science, Vol. 281, No. 5379, 1998, pp. 241-242.

[39] E. E. Escultura, "The Theory of Chaos and Turbulence," In: E. E. Escultura, Ed., Scientific Natural Philosophy, Bentham Science Publishers, Sharjah, 2011, pp. 130-139. doi:10.2174/97816080527071110101

[40] A. Ganeri, “The Ocean Atlas," Dorling Kindersley, London, 1994.

[41] "The Earth Atlas," Dorling Kindersley, London, 1994, pp. 138-139.

[42] E. E. Escultura, "The Big Bang and What It Was," In: J. R. O'Connell and A. L. Hale, Eds., The Big Bang: Theory, Assumptions and Problems, 2012. https://www.novapublishers.com/catalog/product_info.ph p?products_id=21109
[43] G. Schilling, "Watching the Universe's Second Biggest Bang," Science, Vol. 283, No. 5410, 1999, pp. 2013-2014. doi:10.1126/science.283.5410.2003

[44] “The Quasars and Early Galaxies," Scientific American, April 1983, pp. 708-745.

[45] A. Hellemans, "Galaxy's Oldest Stars Shed Light on Big Bang," Science, Vol. 282, No. 5382, 1998, pp. 1428-1429. doi:10.1126/science.281.5382.1428b

[46] E. E. Escultura, "GUT Technology," In: E. E. Escultura, Ed, Scientific Natural Philosophy, Bentham Science Publishers, Sharjah, 2011, pp. 147-148.

http://www.benthamscience.com/ebooks/9781608051786 /index.htm.

[47] E. E. Escultura, "Creative Mathematics Education," Creative Education, Vol. 3, No. 1, 2012, pp. 45-54. http://www.scirp.org/journal/PaperInformation.aspx?pape rID=17266; doi:10.4236/ce.2012.31008

[48] E. E. Escultura, "A Novel Creative Approach to Mathematics-Science Education," In: E. E. Escultura, Ed., Qualitative Math. Modeling: Theoretical and Practical Applications, in Press, LAP LAMBERT Academic Publishing, pp. 229-241. www.lap-publishing.com

[49] E. E. Escultura, "Qualitative Modeling for Complex Systems," Problems of Nonlinear Analysis in Engineering Systems (International IFNA-ANS scientific Journal, Vol. 2, No. 36, 2011, pp. 79-85), Vol. 17, No. 36, 2011, pp. 79-85.

[50] E. E. Escultura, Sustainable Development of the Third World: Focus on the Philippines," Accepted, International Transactions in Operational Research. http://onlinelibrary.wiley.com/journal/10.1111/(ISSN)147 5-3995/issues

[51] E. E. Escultura, "The Origin and Evolution of Biological Species," Journal of Science of Healing Outcomes, No. 6-7, 2010, pp. 17-27.

[52] G. S. Osipenko, A. N. Pokrovsky, B. V. Krylov and V. B. Plakhova, "Mathematical Modeling of Pain Sensation," Proceedings of the Second International Conference on Mathematical Modeling, St. Petersburg, 2000.

[53] V. B. Krylov and B. F. Shehegelov, "Mathematical Methods in the Physiology of Sensory Systems," Second International Conference on Tools for Mathematical Modeling, St. Petersburg, June 1999, pp. 14-19.

[54] E. E. Escultura, "Genetic Alteration, Modification and Sterilization with Applications to the Treatment of Genetic Diseases," The Journal of the Science of Healing Outcomes, No. 8-10, 2011, pp. 30-45. 